\title{
Insights into the Mechanisms That May Clarify Obesity as a Risk Factor for Multiple Sclerosis
}

\author{
Marije J. D. Huitema ${ }^{2} \cdot$ Geert J. Schenk ${ }^{1}$ \\ Published online: 10 March 2018 \\ (C) The Author(s) 2018. This article is an open access publication
}

\begin{abstract}
Purpose of Review The proportion to which genetic and environmental factors contribute to the etiology of multiple sclerosis (MS) is still incompletely understood. An interesting association between MS etiology and obesity has recently been shown although the mechanisms underlying this association are still unknown. We propose deregulated gut microbiota and increased leptin levels as possible mechanisms underlying MS etiology in obese individuals.

Recent Findings Alterations in the human gut microbiota and leptin levels have recently been established as immune modulators in both MS patients and obese individuals. A resemblance between pro-inflammatory bacterial profiles in MS and obese individuals was observed. Furthermore, elevated leptin levels push the immune system towards a more pro-inflammatory state and inhibit the regulatory immune response.

Summary Deregulated gut microbiota and elevated leptin levels may explain the increased risk of developing MS in obese individuals. Further research to confirm causality is warranted.
\end{abstract}

Keywords Obesity $\cdot$ Body mass index $\cdot$ Leptin $\cdot$ Gut microbiota $\cdot$ Multiple sclerosis $\cdot$ Immune system

\section{Introduction}

Multiple sclerosis (MS) is a chronic disease, characterized by demyelination and neurodegeneration in the central nervous system (CNS), resulting in neurological deficits and a wide range of functional disabilities [1]. Worldwide, MS affects approximately 2.3 million people, generally starting in early adulthood. The most common form of MS is relapsingremitting MS (RRMS), which is characterized by multiple lesions in the brain and spinal cord, typically associated with a clinical event and subsequently complete or partial recovery $[2,3]$. Although the cause of MS is still largely unknown, both genetic and environmental factors are involved. To date, the leukocyte antigen HLA-DRB $1 * 15$ allele is the most common

This article is part of the Topical collection on Demyelinating Disorders

Geert J. Schenk

G.Schenk@vumc.nl

1 Department of Anatomy and Neurosciences, Neuroscience Amsterdam, VUmc MS Center Amsterdam, VU University Medical Center, De Boelelaan 1108, 1081 HV Amsterdam, Netherlands

2 Faculty of medicine, VU University, Van der Boechorststraat 7, 1081 BT Amsterdam, Netherlands genetic marker for MS susceptibility [4]. However, individuals carrying this allele may have only a slightly increased risk of developing clinically definite MS [5]. This suggests that additional factors influence disease onset. An interesting environmental factor that has recently been associated with MS etiology is obesity [6]. Due to dietary changes, especially in the western world, obesity has become epidemic. Approximately $35 \%$ of individuals meet criteria for overweight (body mass index (BMI) $25-30 \mathrm{~kg} / \mathrm{m}^{2}$ ) or obesity (BMI $>30 \mathrm{~kg} / \mathrm{m}^{2}$ ) worldwide. Moreover, obesity creates a chronic state of low-grade inflammation, which is possibly associated with an increased risk of developing MS [7-9]. The mechanism responsible for this increased risk of developing MS is still unknown [10]. Two possible hypotheses are discussed in this review.

The first hypothesis suggests that gut microbiota may play a crucial role. The human gut microbiota composition is associated with other metabolic and autoimmune diseases [11]. The gut microbiota consists of the entire genome of microbes in the gut, including bacteria, viruses, and fungi [4]. It plays an essential part in digestion optimization, immune system development, and protection against pathogenic microorganisms [4]. Interestingly, changes in the microbe composition are able to regulate CNS function and vice versa. Several specific 
bacteria have been associated with immune and inflammatory functions in MS patients and might therefore be an important factor for developing MS in genetically predisposed individuals. In addition, alterations in the microbiome are associated with body-weight [10]. If the gut microbiota compositions of MS patients and obese individuals carry significant similarities, and both have differences from non-obese persons, this might explain mechanisms for MS susceptibility in obese individuals.

The second hypothesis involves leptin levels. Leptin is a peptide hormone produced by adipocytes. Its main function is regulating energy expenditure, by monitoring the fat storage and determining food intake via signaling satiety $[12,13]$. Recently, leptin was found to have powerful proinflammatory effects [14]. Moreover, leptin concentrations are positively correlated with BMI, and a link between leptin and MS disease progression has been suggested $[12,15]$. Thus, increased leptin levels may support the association between MS etiology and obesity.

\section{Obesity as a Risk Factor for MS Onset and Its Impact on Disease Progression}

The association between obesity and MS has been investigated extensively. According to a retrospective case-control study, which included $1571 \mathrm{MS}$ cases and 3371 controls, the average weight among MS patients was higher compared with that of their non-MS control subjects during non-diseased adolescence. Furthermore, a BMI above $27 \mathrm{~kg} / \mathrm{m}^{2}$ at age 20 resulted in a twofold increased risk of developing MS. Subjects with a BMI ranging between 25 and $27 \mathrm{~kg} / \mathrm{m}^{2}$ at age 20 also had a slightly increased risk. This association was not found between their current adult BMI and risk of MS [16]. Langer-Gould (2013) stated that an increased risk of developing pediatric MS or clinically isolated syndrome was only observed for obese girls and not for boys, even though obesity was more prevalent in boys [17].

A prospective, longitudinal study reported a similar twofold increase in MS risk when obesity was present during childhood. In addition, pre-puberty overweight and pubertal overweight yielded the same risk of MS. This association was significantly stronger in girls [18].

Moreover, a genetically determined elevated BMI is associated with a $41 \%$ increased risk of MS [19॰]. While obesity in early life may be a risk factor for MS development, it might also relate to an earlier onset of symptoms. According to Kavak et al. (2015), women with overweight and obesity at age 25 had a significantly earlier onset of the disease [20].

Interestingly, in normal-weight subjects, the HLADRB $1 * 15$ allele contributed to a 2.9 -fold increased risk for developing MS. The presence of both obesity and the HLADRB $1 * 15$ allele led to a 9.1 -fold increase in MS risk compared with normal-weight, non-carriers of this allele. These findings strongly support the notion that being obese is an environmental risk factor for developing MS, especially in genetically predisposed individuals [20, 21•]. Interestingly, several studies indicate that despite being a risk factor for acquiring MS, obesity itself does not affect progression of the disease [20, 22-24].

\section{Possible Mechanisms for the Increased Risk of MS Susceptibility in Obese Patients}

\section{The Gut Microbiota}

Animal studies using experimental autoimmune encephalomyelitis (EAE) mice as an MS model show that antibiotics which reduce the gut commensal bacteria impair the development of EAE and reduce EAE severity. This was mainly associated with a reduction of pro-inflammatory cytokines in the circulation and an increase in regulatory $\mathrm{T}$ cells $\left(\mathrm{T}_{\text {reg }}\right)$, which have anti-inflammatory properties. In addition, germ-free mice seem to be resistant to developing EAE or had a milder disease course [25]. In contrast, when mice were colonized with segmented filamentous bacteria, they developed EAE with a more severe disease course. In this case, the CNS contained increased pro-inflammatory Th1 and Th17 cells, suggesting that the gut microbiota are able to mediate immune responses in other tissues [10, 25, 26].

The Gut Microbiota in MS Patients A number of studies have investigated the microbiome in MS patients. Results are summarized in Table 1. Jangi et al. (2016) examined stool samples of 60 RRMS patients and 43 healthy controls (HCs). The results showed significantly increased Methanobrevibacter (phylum Euryarchaeota) and Akkermansia (phylum Verrucomicrobia) genera and decreased Butyricimonas (phylum Bacteroidetes) levels in the feces of patients treated with beta-interferon/glatiramer acetate and untreated MS patients (no treatment for a month) compared with HCs [38•]. From this cohort, patients and HCs with the highest and lowest Methanobrevibacter populations were selected, which resulted in 18 MS patients and 18 HCs. Five hundred sixty-eight immune-related genes from peripheral blood $\mathrm{CD}^{+} \mathrm{T}$ cells and $\mathrm{CD}_{14}{ }^{+}$monocytes were studied. In MS patients, both Methanobrevibacter and Akkermansia were positively correlated with a set of genes expressed in T cells, namely CASP1, TRAF5, and STAT5B [38•]. These genes are involved in interferon (IFN) signaling, interleukin (IL)-2 signaling, and PPAR and RXR pathway activation. The TRAF5 pathway regulates $\mathrm{T}$ cell activation and is known to be overexpressed in MS, as is STAT5B [45]. Butyricimonas was negatively correlated with these genes in T cells, and in HCs, the correlation was nearly zero. Moreover, Butyricimonas positively correlated with the anti-inflammatory cytokine TNFA1P3 and with NFKBIA, which are downregulated in MS [45, 46]. 
Table 1 Summary of the phylum and species of the gut microbiota that are altered in MS patients and obese patients in comparison to healthy controls, showing if species are reduced, increased, or unchanged in the gut microbiota

\begin{tabular}{|c|c|c|c|c|}
\hline Phylum & Bacteria & Immune function & Multiple sclerosis & Obesity \\
\hline Bacteroidetes & Butyricimonas & $\begin{array}{l}\text { Anti-inflammatory } \\
\text { (butyrate-producing) [1] }\end{array}$ & $\downarrow[25], \downarrow[27], \downarrow[28]^{*}$ & \\
\hline Obesity: $\downarrow$ & Prevotella & & $\downarrow[25], \downarrow[29], \downarrow[30], \uparrow[28]^{*}$ & $\uparrow[31], \leftrightarrow[32 \cdot \bullet], \leftrightarrow[33]$ \\
\hline$[34,35]$ & Bacteroides & & $\downarrow[29]$ & $\downarrow[32 \bullet \bullet], \downarrow[28]$ \\
\hline \multirow[t]{3}{*}{ MS: $\downarrow[30]$} & Parabacteroides & & $\downarrow[30]$ & \\
\hline & Flavobacterium & & $\uparrow[30]$ & \\
\hline & Pedobacter & & $\uparrow[30]$ & \\
\hline Euryarchaeota & $\begin{array}{l}\text { Methanobrevibacter } \\
\quad \text { (smithii) }\end{array}$ & Pro-inflammatory [1] & $\uparrow[25], \uparrow[27], \uparrow[28]^{*}$ & $\uparrow[36], \uparrow[31], \leftrightarrow[37]^{*}, \downarrow[35]$ \\
\hline Verrucomicrobia & Akkermansia & Pro-inflammatory [1] & $\uparrow[25], \uparrow[27], \uparrow[28]^{*}$ & $\downarrow[31], \downarrow[27]$ \\
\hline Firmicutes & $\begin{array}{l}\text { Faecalibacterium } \\
\text { Prausnitzii }\end{array}$ & $\begin{array}{l}\text { Anti-inflammatory } \\
\text { (butyrate-producing) [2] }\end{array}$ & $\downarrow[29] \leftrightarrow[30]$ & $\uparrow[33]$ \\
\hline \multirow[t]{9}{*}{ Obesity: $\uparrow[32 \bullet \bullet]$} & $\begin{array}{l}\text { Clostridia cluster } \\
\text { XIVa and IV }\end{array}$ & $\begin{array}{l}\text { (Anti-inflammatory: some are } \\
\text { butyrate-producing) [5] }\end{array}$ & $\downarrow[29]$ & \\
\hline & $\begin{array}{l}\text { Clostridium perfringens } \\
\quad(\text { cluster I) }\end{array}$ & & - & $\leftrightarrow[32 \bullet \bullet]$ \\
\hline & Streptococcus & & $\uparrow[29]$ & \\
\hline & Lactobacillus & & $\downarrow[30], \uparrow[28]^{*}$ & $\leftrightarrow[32 \bullet \bullet] \leftrightarrow[33], \uparrow[37]^{*}, \uparrow[35]$ \\
\hline & Erysipelotrichaceae & $\begin{array}{l}\text { (Anti-inflammatory: some are } \\
\text { butyrate-producing) [5] }\end{array}$ & $\downarrow[30]$ & $\uparrow[31]$ \\
\hline & Blautia & & $\uparrow[30], \uparrow[28]^{*}$ & \\
\hline & Dorea & & $\uparrow[30], \downarrow[28]^{*}$ & $\uparrow[28]$ \\
\hline & Anaerostipes & & $\downarrow[29]$ & \\
\hline & Lachnospiraceae & & $\downarrow[25], \downarrow[30], \downarrow[28]^{*}$ & $\uparrow[28]$ \\
\hline \multirow[t]{4}{*}{ Actinobacteria } & Collinsella & & $\downarrow[25], \downarrow[30]$ & \\
\hline & Slackia & & $\downarrow[25]$ & \\
\hline & Bifidobacterium & & $\uparrow[29], \uparrow[28]^{*}$ & $\leftrightarrow[32 \bullet \bullet], \leftrightarrow[33], \downarrow[37]^{*}$ \\
\hline & Adlercreutzia & & $\downarrow[30]$ & \\
\hline \multirow[t]{4}{*}{ Proteobacteria } & Pseudomonas & & $\uparrow[30]$ & \\
\hline & Mycoplana & & $\uparrow[30]$ & \\
\hline & Sutterella & & $\downarrow[29], \uparrow-$ therapy [25] & \\
\hline & Haemophilus & & $\downarrow[30]$ & \\
\hline
\end{tabular}

References: [38•]. Jangi et al. (2016), [39]. Miyake et al. (2015), [40••]. Chen et al. (2016), [32••]. Mbakwa et al. (2015), [33]. Zhang et al. (2009), [34]. Zuo et al. (2011) [41]. Balamurugan et al. (2010) [42]. Ley (2006). [27]. Cantarel et al. (2015) [28]. Tremlett (2016), [37]. Yun et al. (2017), [35]. Liu et al. (2017), [43]. Ignacio et al. (2015), [44]. Million et al. (2013)

*Pediatric

In monocytes of MS patients, Methanobrevibacter and Akkermansia were also positively correlated with several genes, namely MAPK14, MAPK1, LTBR, STAT5B, CASP1, and HLA-DRB1. Whereas Butyricimonas had negative correlations with these genes. They are involved in the maturation of dendritic cells (DCs) and INF signaling. MAPK genes in monocytes can activate the immune system and play a role in MS pathogenesis [45]. In untreated MS patients, Akkermansia was positively correlated, and Butyricimonas was negatively correlated with these genes in monocytes, while these correlations were not found in HCs. This suggests that increased levels of Methanobrevibacter and Akkermansia might have proinflammatory properties in MS patients, by activating DCs and recruitment of inflammatory cells [47,
48]. A reduced Butyricimonas level was also associated with pro-inflammatory gene expression and with less butyrate production [38]. Butyrate normally activates colonic $T_{\text {reg }}$ cells, inhibits the inflammatory IL-12, and activates the antiinflammatory IL-10 in monocytes, which promotes an antiinflammatory state. Thus, less butyrate production may disrupt gut barrier function and induce inflammation [29, 49].

Miyake et al. (2015) followed a cohort of Japanese HCs and RRMS patients during remission. The phyla Firmicutes, Bacteroidetes, Proteobacteria, and Actinobacteria were the major phyla present for both MS patients and HCs. However, the Bifidobacterium and Streptococcus genera were more abundant, and 19 species were less abundant in MS patients, with 14 of them belonging to Clostridia clusters IV and XIV (phylum 
Firmicutes). These Clostridia species contain butyrateproducing bacteria [30, 39]. Additionally, several Bacteroides (phylum Bacteroidetes), Faecalibacterium prausnitzii, and Anaerostipes were less abundant in MS patients. Faecalibaterium prausnitzii belongs also to the butyrate-producing species [50]. Consistent with Jangi et al. (2016), a decrease in Prevotella (phylum Bacteroidetes) was observed, which is negatively correlated with MS pathogenesis [38 $]$.

Finally, Chen et al. (2016) compared RRMS patients during remission and active phases to HCs. While the overall species richness did not significantly differ between MS and $\mathrm{HCs}$, there was a significant difference between active MS patients and MS patients in remission. Importantly, the gut microbiota composition of patients in remission resembled that of the HCs $\left[40^{\circ} \cdot\right.$. An abundance of the genus Blautia (phylum Firmicutes) in MS patients and an abundance of Parabacteroides (phylum Bacteroidetes) in HCs were observed, which implies that MS patients have a gut microbial dysbiosis. Additionally, a prediction model was used, which was consistent with the microbes found with 16S rRNA gene sequencing. Findings are summarized in Table $1[40 \bullet \bullet]$. Notably, Prevotella is reduced and the sub-type Prevotella histicola is known to suppress EAE [51]. Erysipelotrichaceae is also decreased and has anti-inflammatory effects, by playing a major role in the bile gut metabolism $[31,36]$. This suggests that decreased levels of these species play a role in disease exacerbation $[40 \bullet \cdot]$.

In summary, depletion or enrichment of specific species in the gut microbiota establishes either pro-inflammatory or antiinflammatory effects and may thus have substantial impact on MS pathogenesis.

The Gut Microbiota in Obese Patients To evaluate a possible parallel with MS patients, the microbiota of obese individuals is evaluated below. Findings are summarized in Table 1.

Mbakwa et al. (2015) examined 472 children and quantified Methanobrevibacter smithii and Methanobrevibacter stadtmanae, because these were previously associated with overweight. M. smithii was present in 369 and M. stadtmanae in 39 children. M. stadtmanae was not significantly associated with overweight. However, when adjusting for confounders, children with a high count of $M$. smithii were three times more likely to be overweight [32••].

This is in line with Zhang et al. (2009), who observe increased methanogenic Archaea, including M. smithii, in obese individuals. In addition, Prevotellaceae was significantly increased, and Akkermansia was reduced in obese individuals compared with normal-weight subjects [33].

Other studies have found conflicting correlations. In a Chinese study of obese subjects $(N=52)$, the level of Bacteroides and Clostridum perfringens ( $C$. perfringens) was significantly lower than in normal-weight subjects $(N=$ 52) [34]. A study done by Balamurugan et al. (2010) observed no differences in amount of Lactobacillus and Bifidobacterium in obese and normal-weight Indian children. Moreover, no significant difference in Prevotella amount was observed [41].

Also, a reduced amount of the phylum Bacteroidetes and an increased amount of the phylum Firmicutes were observed in obese subjects, which are normally beneficial bacteria dominating in the human gut. When obese patients were assigned to a low-calorie diet, the proportion Bacteroidetes increased simultaneously with weight loss. This implies that the phylum Bacteroidetes is negatively correlated with weight [42].

Le Chatelier et al. (2013) divided individuals into low-gene count (LGC) and high-gene count (HGC) groups. The LGC group represented $23 \%$ of the study population and contained a significantly higher level of obese individuals. In addition, LGC obese individuals had lower microbial diversity compared with the HCG individuals. This was associated with a decrease in eight butyrate-producing species in the gut microbiota of LGC individuals. M. smithii and Faecalibacterium were also decreased. Low bacterial genetic diversity was associated with an increased serum leptin, decreased serum adiponectin, more insulin resistance, and a more marked inflammatory phenotype. This low-grade inflammation and insulin resistance could be due to the reduced amount of butyrate-producing species, which might shift the immune system towards a more inflammatory state.

\section{The Role of Leptin in the Immune System}

The mechanism that may explain the association between MS pathogenesis and obesity is the presence of significantly increased levels of the adipose tissue hormone leptin in both patient populations [21, 52, 53].

Leptin plays a role in innate and adaptive immunity. The adipokines (leptin, adiponectin, and pro-inflammatory cytokines) are secreted in the circulation and connect the adipose and lymphoid tissue compartments. Adipose tissue differentiation and leptin secretion can be induced by the proinflammatory cytokines, IL-1, and tumor-necrosis factor (TNF). Leptin release supports the differentiation of proinflammatory $T$ helper $1\left(\mathrm{~T}_{\mathrm{H}} 1\right)$ cells. In the presence of leptin, a higher proliferation rate and production of IL- 2 is mediated in naïve T cells. However, minimal proliferation of memory $\mathrm{T}$ cells is induced, which results in increased production of interferon (INF)- $\gamma$. In turn, INF- $\gamma$ is able to induce $\mathrm{T}_{\mathrm{H}} 1$ responses. Moreover, leptin can act as an early acute phase reactant, similar to C-reactive protein (CRP), IL-6, and IL-1, which are released in high amounts during bacterial infection, sepsis, and inflammation [13].

Leptin acts on the leptin receptor (LEPR) and is expressed by $\mathrm{CD}^{+}, \mathrm{CD} 8^{+}$human $\mathrm{T}$ cells, $\mathrm{T}_{\text {reg }}$ cells, B cells, monocytes/ macrophages, endothelial cells, and natural killer (NK) cells. [13]. LEPR belongs to the class I cytokine receptor family and 
is therefore able to activate the signaling proteins JAK (Janus Kinase) and STAT (signal transducer and activator of transcription). Subsequently, JAK2 tyrosine kinase will be activated, which in turn activates STAT3. Also, the phosphorylation of STAT3 in $\mathrm{CD}^{+}, \mathrm{CD} 25^{-}$effector T cells is mediated by leptin. On the contrary, there are no associations with induction of STAT3 phosphorylation in $\mathrm{CD} 4^{+}, \mathrm{CD} 25^{+} \mathrm{T}_{\text {reg }}$ cells, which confirms their hypo-responsiveness. $\mathrm{T}_{\text {reg }}$ cells are a subset of the $\mathrm{CD}^{+} \mathrm{T}$ cell that regulate immune suppression and maintain self-tolerance. $T_{\text {reg }}$ cells are characterized by promoting Foxp3 (forkhead family transcription factor $\mathrm{p} 3$ ) and increased levels of the IL-2 receptor (CD25) [7, 54]. Neutralizing leptin levels results in a reversal of inactivity and reduced proliferation of $\mathrm{T}_{\text {reg }}$ cells $[12,55]$. This is in line with findings described by Moraes-Vieira et al. (2013), who observed that $\mathrm{CD}^{+} \mathrm{T}$ cells of leptin-deficient mice have an increased generating capacity of $\mathrm{T}_{\text {reg }}$ cells [56]. Figure 1 summarizes the most important effects of leptin.

MS and Leptin Considering leptin as a serious pro-inflammatory modulator, its effects might explain the association between obesity and MS, since leptin is also increased in MS patients [53, 57]. Interestingly, it has been shown that leptin plays a key role in
EAE by proliferation of $\mathrm{CD}^{+}{ }^{+}$autoreactive $\mathrm{T}$ cells. Leptin-deficient $\left(\right.$ Lep $\left.^{\text {ob/ob }}\right)$ mice injected with transferred encephalitogenic MOG35-55-specific CD4 ${ }^{+} \mathrm{T}$ cells failed to develop EAE. Moreover, this coincided with reduced pro-inflammatory cytokines (IL-2, IL-6, INF- $\gamma$, TNF- $\alpha$ ) and a reduced cell proliferation of transferred MOG35-55-specific $\mathrm{CD}^{+}{ }^{+} \mathrm{T}$ cells. In contrast, the MOG35-55-specific CD4 ${ }^{+} \mathrm{T}$ cells did induce EAE in Lep ${ }^{\text {ob/ob }}$ mice with leptin replacement. Treatment with leptin also restored the reduced proliferation and pro-inflammatory cytokine levels in Lep $^{\mathrm{ob} / \mathrm{ob}}$ mice [58]. Moreover, R-EAE mice (a model for RRMS) with lowered leptin levels showed a better clinical outcome, less relapses, and a reduced disease progression [15].

In MS patients, Emamgholipour et al. (2013) observed that leptin is negatively correlated with FoxP3 gene expression in PBMCs and is positively correlated with TNF- $\alpha$, IL- $1 \beta$, and hsCRP levels [59]. In addition, during the course of MS, leptin concentrations were significantly increased in female patients $[60,61]$. These elevated levels can create a positive feedback loop, which leads to MS progression: higher leptin levels cause a reduction of Foxp3 expression and increased production of pro-inflammatory cytokines. These cytokines lower Foxp3 even more, which in turn cause an increase of leptin and pro-inflammatory cytokines [59].

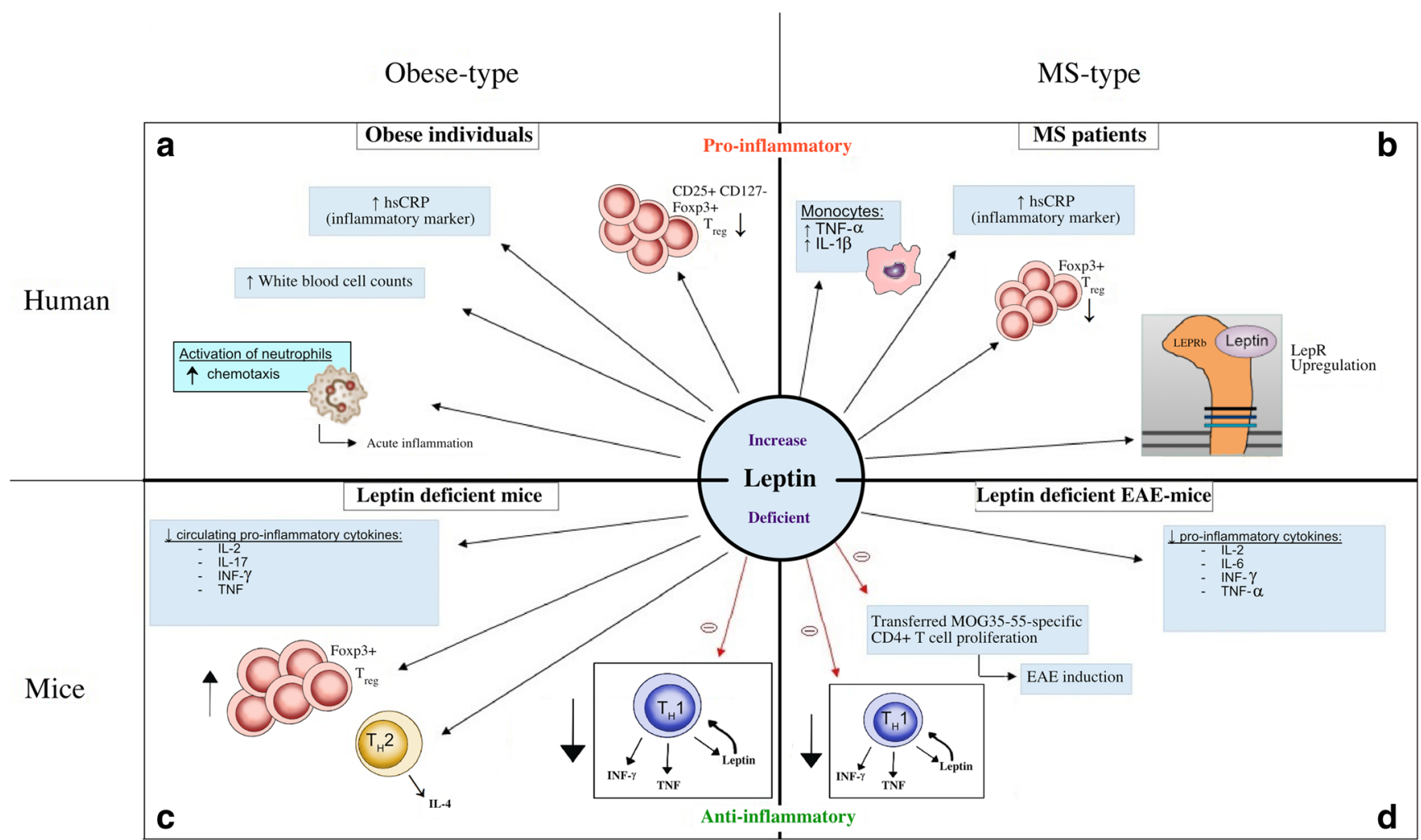

Fig. 1 Summary of the effects of leptin in human and mice. Leptin affects the immune system in both mice and human. a In MS patients, an increase in leptin causes an upregulation of pro-inflammatory cytokines, a decrease in the anti-inflammatory Foxp $3+\mathrm{T}_{\text {reg }}$ cells, and an upregulation of the LepR. b Similarly, in obese individuals, a decrease in Foxp3 $+T_{\text {reg }}$ cells exists. Moreover, they show an increase in white blood cells and activated neutrophils, which puts the immune system more towards a pro-inflammatory state and induces acute inflammation. c In leptin-deficient mice, a decrease in pro-inflammatory cytokines and an increase in Foxp3 $+T_{\text {reg }}$ cells are present. d Moreover, EAE (induced by MOG35-55-specific CD4+ T cells) is not able to develop when leptin is not present 
A leptin increase was observed during the acute phase of the disease in both the CSF and serum of untreated RRMS patients compared to the remitting phase, in contrast with the control group. This is possibly secondary to in situ synthesis of leptin in the CNS or increased transport across the bloodbrain barrier upon enhanced systemic production. This was positively correlated with increased INF- $\gamma$ production in the CSF and inversely correlated with the percentage of circulating $\mathrm{T}_{\text {reg }}$ cells [57].

Elevated concentrations of leptin have been found in MS patients [62], although Rotondi et al. (2013) found similar leptin levels in patients and healthy controls [61].

In RRMS relapsing patients, an upregulation of the phosphorylated STAT3 in $\mathrm{CD}^{+}, \mathrm{CD}^{+} \mathrm{T}$ cells, and PBMCs was found. This leads towards upregulation of the leptin receptor in the acute phase of the disease and enhances leptin sensitivity in RRMS patients. These data suggest that the LEPR, rather than leptin itself, might play a role in MS pathogenesis in the acute phase of the disease by upregulating the immune response [63]. This is supported by the finding that no difference in leptin concentration was found between the pre-relapse and remission phases in patients naïve to treatment [62].

Obesity and Leptin The association between obesity and a low-grade inflammatory state of the adipose tissue has frequently been investigated [7, 64]. Wagner et al. (2013) compared non-obese with obese subjects. They isolated PBMCs and found a reduced amount of circulating CD25 ${ }^{+} \mathrm{CD} 127 \mathrm{Foxp}^{+}$ $\mathrm{T}_{\text {reg }}$ cells in obese individuals, and this correlated with body weight, BMI, and circulating leptin levels. Furthermore, a significant reduction of $\mathrm{T}_{\text {reg }}$ cells was also found in individuals that had elevated systemic inflammatory markers (hsCRP) or increased HbA1c (a marker for impaired glucose tolerance), which mainly occurred in obesity [7]. This was in accordance with an observational cohort of adolescents. Obese individuals had increased levels of hsCRP and elevated white blood cell counts, which suggests a chronic inflammatory state. Additionally, high circulating neutrophil numbers were observed in obese individuals, indicating acute inflammation [65].

In a study in rodents, Moraes-Vieira et al. (2013) examined the effects of leptin and observed lower circulating proinflammatory cytokines (TNF, INF- $\gamma$, IL-2, and IL-17) in Lep $^{\text {ob/ob }}$ mice compared with controls. Moreover, they observed a shift towards an anti-inflammatory $\mathrm{Th}_{2} \mathrm{~T}$ cell response and an increase in Foxp $3 \mathrm{~T}_{\text {reg }}$ cells, and CD4 ${ }^{+} \mathrm{T}$ cells showed a reduced alloreactivity [56]. Above all, when administering leptin to Lep ${ }^{\mathrm{ob} / \mathrm{ob}}$ mice, leptin induces suppression of the anti-inflammatory $\mathrm{Th}_{2}$ response and activates the $\mathrm{Th}_{1} \mathrm{im}$ mune response, by increasing the production of proinflammatory cytokines (INF- $\gamma$ and IL-2) $[15,66]$. This, together with a reduction in $\mathrm{T}_{\text {reg }}$ cells, sustains the plausibility of pro-inflammatory effects of leptin in obese individuals.

\section{Discussion}

The aim of this review is to describe the impact of obesity on MS onset and to highlight the potential mechanisms that may underlie MS etiology in obese patients. To this end, we asked if (1) changes in the composition of the gut microbiota or (2) altered leptin levels are possible explanations for the increased risk of developing MS in obese individuals. We hypothesized that the gut microbiome composition of obese individuals resembles that of MS patients and might therefore be an explanation for the increased risk of MS development.

While strong associations are found between early life obesity and an increased risk of developing MS [16-18, 19•, 20, 21], no associations are found between obesity and disease progression [20, 23, 24].

Similarities between some, but not all, gut microbes were observed in MS patients and obese individuals. An overall reduction in the phylum Bacteroidetes in obese individuals was found, which resembles the overall observed reduction in this phylum for MS patients [40••, 42, 44]. Specifically the bacteria Bacteroides in this phylum were reduced in both MS and obese subjects. Moreover, the presence of Bacteroides fragilis is known to coincide with disease attenuation in EAE. Its capsular polysaccharide $\mathrm{A}$ is associated with reducing disease severity, by mediating elevated numbers of FoxP3 $+\mathrm{T}_{\text {reg }}$ cells in the cervical lymph nodes [4, 67]. Thus, a reduced number of Bacteroides in obese individuals might contribute to MS susceptibility, by pushing the immune system towards a more inflammatory state.

In the phylum Euryarchaeota, the bacterium M. smithii is increased in the gut microbiota of both MS patients and obese individuals. Due to its pro-inflammatory properties, T cells and DCs are activated, which are both correlated with MS pathogenesis. Therefore, elevated levels of $M$. smithii support the MS/obesity microbiome hypothesis.

In contrast, no consensus was found between the microbes Akkermansia, Faecalibacterium prausnitzii, Lactobacillus, and Prevotella. In fact, the amount of Akkermansia, which is pro-inflammatory and is positively correlated with MS pathogenesis, was reduced in obese individuals. The same applies for the anti-inflammatory bacteria Faecalibacterium prausnitzii and Erysipelotrichaceae, which are decreased in MS and increased in obesity. In addition, a significant reduction of Prevotella is observed in MS patients, which is correlated with MS pathogenesis, whereas both an increased amount and no changes were observed in obese individuals $[33,39,40 \bullet \bullet, 41]$. This suggests that Prevotella is not associated with the increased risk of MS in obese individuals. A possible explanation could be the increased intake of carbohydrates in diets leading to an increase of Prevotella. Besides variation in diets, there are more possible confounders that might influence the gut microbiota, for instance red wine and aspartame consumption [68]. However, not all included 
studies have been corrected for food consumption. The modulation of MS and EAE by dietary interventions was recently reviewed extensively [69]. Interestingly, immunomodulatory therapy was associated with increased amounts of Prevotella and Sutterella in treated MS patients, compared with untreated MS patients and HCs. This implies that immunomodulatory treatment may establish a more normalized gut microbiota composition, at least for some microbes [38•], further underscoring a causal role for gut microbiota in MS.

Longitudinal study designs in larger patient and control groups are necessary to provide suitable clinical, pathological, and genetic data to further investigate the interplay of obesity and MS. If causality indeed exists between obesity and MS, prevention of obesity during childhood will establish a $15 \%$ reduction in the number of new MS cases [6]. This offers significant prospects for lifestyle interventions and the use of selective anti- or probiotics to normalize the gut microbiota composition.

The second hypothesis we considered was that increased levels of the fat-derived hormone, leptin contribute to the development of MS in obese individuals, since leptin levels are increased in both obese people and MS patients [21, 52, 53]. Specifically high amounts of leptin were present in the CSF and sera of MS patients and significantly more in the CSF during relapses [15], which is possibly due to synthesis of leptin in the CNS or an increased transport across the blood-brain barrier. This implicates that leptin plays a key role in the inflammation that is taking place in the brain during the course of MS.

Moreover, leptin is capable of reducing $\mathrm{T}_{\text {reg }}$ cells, inducing neutrophil activation, and increasing white blood cell counts and hsCRP. This could implicate that obese individuals might be more susceptible to developing MS, because their immune system is more skewed towards a pro-inflammatory state.

Caloric restriction seems to increase survival and life span rates in EAE mice. It ameliorates the disease, reduces inflammation, and reduces leptin levels. However, no suppression of the immune system was found in this case. This might suggest a possible lifestyle intervention option for MS, but hitherto little research has been done in humans [12].

More interestingly, female MS patients showed higher serum leptin levels than males, despite having a similar BMI. This gender effect was present in both healthy controls and MS patients. This might partly explain the fact that women are more likely to develop MS than men, with a ratio of almost 3:1 $[63,70]$.

\section{Conclusion}

In conclusion, alterations in the gut microbiome of obese individuals show similarities to those of MS patients. Alterations in abundance of the microbes M. smithii, Bacteroides, and in the phylum Bacteroidetes might put the immune system towards a more inflammatory state. The phylum Bacteroidetes showed the most consistent results in this regard. Therefore, the hypothesis that changes in the composition of the gut microbiota are a possible explanation for the increased risk of developing MS in obese individuals is confirmed for some, but not all phyla. Alterations in some genera did not correspond between MS patients and obese individuals, and this review only defines a possible association, thus further research to confirm causality is warranted.

In light of the hypothesis that altered leptin levels are an explanation for the increased risk of developing MS in obese individuals, we conclude that leptin is a modulating factor that switches the immune system towards a more proinflammatory state, by secretion of pro-inflammatory cytokines, upregulation of the leptin receptor, and downregulation of anti-inflammatory $\mathrm{T}_{\text {reg }}$ cells. Therefore, leptin and leptin receptor levels might be a plausible factor contributing to the increased risk of MS in obese individuals.

Finally, in individuals with a low bacterial genetic gut diversity, more weight gain and higher amounts of leptin were found [71]. This suggests that not only alterations in the gut microbiome composition but also increased levels of leptin are present in obese individuals, together contributing to MS susceptibility.

Acknowledgements We would like to lend special thanks to Dr. AnneMarie van Dam for providing critical comments.

\section{Compliance with Ethical Standards}

Conflict of Interest Marije J.D. Huitema and Geert J. Schenk declare no conflict of interest.

Human and Animal Rights and Informed Consent This article does not contain any studies with human or animal subjects performed by any of the authors.

Open Access This article is distributed under the terms of the Creative Commons Attribution 4.0 International License (http:// creativecommons.org/licenses/by/4.0/), which permits unrestricted use, distribution, and reproduction in any medium, provided you give appropriate credit to the original author(s) and the source, provide a link to the Creative Commons license, and indicate if changes were made.

\section{References}

Papers of particular interest, published recently, have been highlighted as:

- Of importance,

• Of major importance

1. Harbo HF, Gold R, Tintoré M. Sex and gender issues in multiple sclerosis. Ther Adv Neurol Disord. 2013;6(4):237-48. 
2. "National Multiple Sclerosis Society: Who gets MS?," New York: National MS Society, 2011. [Online]. Available: http://www. nationalmssociety.org/What-is-MS/Who-Gets-MS. [Accessed: 30Dec-2016].

3. Stys PK, Zamponi GW, van Minnen J, Geurts JJG. Will the real multiple sclerosis please stand up. Nat Rev Neurosci. 2012;13(7): 507-14.

4. D. W. Mielcarz and L. H. Kasper, "The Gut Microbiome in Multiple Sclerosis," Curr Treat Options Neurol, vol. 17, no. 4. 2015.

5. Goodin DS. The causal cascade to multiple sclerosis: a model for MS pathogenesis. PLoS One. 2009;4(2):e4565.

6. Ascherio A, Munger K. Epidemiology of multiple sclerosis: from risk factors to prevention. Semin Neurol. 2016;36(1):103-14.

7. Wagner $\mathrm{NM}$, et al. Circulating regulatory $\mathrm{T}$ cells are reduced in obesity and may identify subjects at increased metabolic and cardiovascular risk. Obesity. 2013;21(3):461-8.

8. Munger KL, Chitnis T, Ascherio A. Body size and risk of MS in two cohorts of US women. Neurology. 2009;73(19):1543-50.

9. de Guerrero-García J, Carrera-Quintanar L, López-Roa RI, Márquez-Aguirre AL, Rojas-Mayorquín AE, Ortuño-Sahagún D. Multiple sclerosis and obesity: possible roles of adipokines. Mediat Inflamm. 2016;2016:1-24.

10. Bhargava P, Mowry EM. Gut Microbiome and Multiple Sclerosis. Curr Neurol Neurosci Rep. 2014;14:492.

11. E. M. M. Quigley, "Gut bacteria in health and disease.," Gastroenterol Hepatol. (N. Y)., vol. 9, no. 9, pp. 560-569, 2013.

12. Matarese G, Carrieri PB, Montella S, De Rosa V, La Cava A. Leptin as a metabolic link to multiple sclerosis. Nat Rev Neurol. 2010;6(8): 455-61.

13. La Cava A, Matarese G. The weight of leptin in immunity. Nat Rev Immunol. 2004;4(5):371-9.

14. Procaccini C, La Rocca C, Carbone F, De Rosa V, Galgani M, Matarese G. Leptin as immune mediator: interaction between neuroendocrine and immune system. Dev Comp Immunol. 2017;66: $120-9$.

15. Matarese G, et al. Leptin increase in multiple sclerosis associates with reduced number of CD4+CD25+ regulatory T cells. Proc Natl Acad Sci. 2005;102(14):5150-5.

16. Hedstrom AK, Olsson T, Alfredsson L. High body mass index before age 20 is associated with increased risk for multiple sclerosis in both men and women. Mult Scler J. 2012;18(9):1334-6.

17. Langer-Gould A, Brara SM, Beaber BE, Koebnick C. Childhood obesity and risk of pediatric multiple sclerosis and clinically isolated syndrome. Neurology. 2013;80:548-52.

18. Munger KL, et al. Childhood body mass index and multiple sclerosis risk: a long-term cohort study. Mult Scler J. 2013;19(10): 1323-9.

19. L. E. Mokry, S. Ross, N. J. Timpson, S. Sawcer, G. Davey Smith, and J. B. Richards, "Obesity and Multiple Sclerosis: A Mendelian Randomization Study," PLoS Med., vol. 13(6), p. e1002053, 2016. Showing a significant association between a genetically determined elevated BMI and an increased risk of developing MS.

20. Kavak K, Teter B, Hagemeier J, Zakalik K, Weinstock-Guttman B. Higher weight in adolescence and young adulthood is associated with an earlier age at multiple sclerosis onset. Mult Scler J. 2015;21(7):858-65.

21. Hedström AK, et al. Interaction between adolescent obesity and HLA risk genes in the etiology of multiple sclerosis. Neurology. 2014;82:826-72. Showing a high impact of having the HLADRB1*15 allele and being obese, compared with non-obese

22. Tettey P, et al. Adverse lipid profile is not associated with relapse risk in MS: results from an observational cohort study. J Neurol Sci. 2014;340:230-2.
23. Bove R, et al. Longitudinal BMI trajectories in multiple sclerosis: sex differences in association with disease severity. In: Mult. Scler. Relat. Disord, vol. 8: Elsevier; 2016. p. 136-40.

24. Çoban A, Çevik D, Özyurt S, Gencer M, Tüzün E, Türkoğlu R. The association between obesity and oligoclonal band formation in multiple sclerosis patients. In: Obes. Res. Clin. Pract, vol. 9: Elsevier; 2015. p. 533-5.

25. Ochoa-Repáraz J, Mielcarz DW, Ditrio LE, Kasper LH. Role of Gut Commensal Microflora in the Development of Experimental Autoimmune Encephalomyelitis. J Immunol. 2009;183(10):6041-50.

26. Lee YK, Menezes JS, Umesaki Y, Mazmanian SK. Proinflammatory T-cell responses to gut microbiota promote experimental autoimmune encephalomyelitis. Proc Natl Acad Sci. 2011;108(Supplement 1):4615-22.

27. Cantarel BL, et al. Gut microbiota in MS: possible influence of immunomodulators. J Investig Med. 2015;63(5):729-34.

28. Tremlett $\mathrm{H}$, et al. Gut microbiota in early pediatric multiple sclerosis: a case-control study. Eur J Neurol. 2016;23(8):1308-21.

29. Y. Furusawa et al., "Commensal microbe-derived butyrate induces the differentiation of colonic regulatory T cells," Nature, vol. 0 , 2013.

30. Atarashi $\mathrm{K}$, et al. Treg induction by a rationally selected mixture of Clostridia strains from the human microbiota. Nature. 2013;500(7461):232-6.

31. Vavassori P, Mencarelli A, Renga B, Distrutti E, Fiorucci S. The bile acid receptor FXR is a modulator of intestinal innate immunity. J Immunol. 2009;183(10):6251-61.

32.• Mbakwa CA, et al. Gut colonization with methanobrevibacter smithii is associated with childhood weight development. Obesity. 2015;23:2508-16. This paediatric study examined the proinflammatory bacteria, methanobrevibacter smithii

33. Zhang $\mathrm{H}$, et al. Human gut microbiota in obesity and after gastric bypass. PNAS. 2009;106:2365-70.

34. Zuo H-J, Xie ZM, Zhang WW, Li YR, Wang W, Ding XB, et al. Gut bacteria alteration in obese people and its relationship with gene polymorphism Gut bacteria alteration in obese people and its relation- ship with gene polymorphism. World J Gastroenterol. 2011;17(8):1076-81.

35. R. Liu et al., "Gut microbiome and serum metabolome alterations in obesity and after weight-loss intervention," Nat Med, vol. advance on, 2017.

36. Labbé A, Ganopolsky JG, Martoni CJ, Prakash S, Jones ML. Bacterial bile metabolising gene abundance in Crohn's, ulcerative colitis and type 2 diabetes metagenomes. PLoS One. 2014;9(12):117.

37. Yun Y, et al. Comparative analysis of gut microbiota associated with body mass index in a large Korean cohort. BMC Microbiol. 2017;17(1):151

38. S. Jangi et al., "Alterations of the human gut microbiome in multiple sclerosis," Nat Commun., vol. 7, p. 12015, Jun. 2016. Found an altered variety of gut microbal genera in treated and untreated MS patients, compared with healthy controls.

39. Miyake S, et al. Dysbiosis in the Gut Microbiota of Patients with Multiple Sclerosis, with a Striking Depletion of Species Belonging to Clostridia XIVa and IV Clusters. PLoS One. Sep. 2015;10(9): e0137429.

40.• J. Chen et al., "Multiple sclerosis patients have a distinct gut microbiota compared to healthy controls," Sci Rep., vol. 6, no. 1, p. 28484, Sep. 2016. This study provides an extensive number of gut microbiota species, comparing RRMS with age- and gender matched healthy controls.

41. Balamurugan R, et al. Quantitative differences in intestinal Faecalibacterium prausnitzii in obese Indian children. $\mathrm{Br} J$ Nutr. Feb. 2010;103(3):335-8. 
42. Ley RE, Turnbaugh PJ, Klein S, Gordon JI. Microbial ecology: Human gut microbes associated with obesity. Nature. 2006;444: $1022-3$.

43. Ignacio A, et al. Correlation between body mass index and fecal microbiota from children. Clin Microbiol Infect. 2015;22(3):1-8.

44. Million M, et al. Correlation between body mass index and gut concentrations of Lactobacillus reuteri, Bifidobacterium animalis, Methanobrevibacter smithii and Escherichia coli. Int J Obes. 2013;3720:1460-6.

45. Achiron A, Gurevich M, Friedman N, Kaminski N, Mandel M. Blood transcriptional signatures of multiple sclerosis: unique gene expression of disease activity. Ann Neurol. 2004;55(3):410-7.

46. F. Gilli et al., "Learning from nature: pregnancy changes the expression of inflammation-related genes in patients with multiple sclerosis," PLoS One, vol. 5, no. 1, p. e8962, 2010.

47. Bang C, Weidenbach K, Gutsmann T, Heine H, Schmitz RA. The intestinal archaea methanosphaera stadtmanae and methanobrevibacter smithii activate human dendritic cells. PLoS One. 2014;9(6):e99411.

48. Derrien $\mathrm{M}$, et al. Modulation of mucosal immune response, tolerance, and proliferation in mice colonized by the mucin-degrader Akkermansia muciniphila. Front Microbiol. 2011;2(166):1-14.

49. Säemann MD, et al. Anti-inflammatory effects of sodium butyrate on human monocytes: potent inhibition of IL-12 and up-regulation of IL-10 production. FASEB J. 2000;14(15):2380-2.

50. Machiels K, et al. A decrease of the butyrate-producing species Roseburia hominis and Faecalibacterium prausnitzii defines dysbiosis in patients with ulcerative colitis. Gut. 2014;63:1275-83.

51. M. Murri et al., "Gut microbiota in children with type 1 diabetes differs from that in healthy children: a case-control study," $B M C$ Med, vol. 11, no. 46, 2013.

52. Maffei M, et al. Leptin levels in human and rodent: measurement of plasma leptin and ob RNA in obese and weight-reduced subjects. Nat Med. 1995;1(11):1155-61.

53. Kraszula Ł, Jasińska A, Eusebio M-O, Kuna P, Głąbiński A, Pietruczuk M. Evaluation of the relationship between leptin, resistin, adiponectin and natural regulatory $\mathrm{T}$ cells in relapsingremitting multiple sclerosis. Neurol Neurochir Pol. 2012;46(1): 22-8.

54. Berg AH, Scherer PE. Adipose tissue, inflammation, and cardiovascular disease. Circ Res. 2005;96(9):939-49.

55. De Rosa $\mathrm{V}$, et al. A key role of leptin in the control of regulatory $\mathrm{T}$ cell proliferation. Immunity. 2007;26(2):241-55.

56. Moraes-Vieira PMM, et al. Leptin modulates allograft survival by favoring a Th2 and a regulatory immune profile. Am J Transplant. 2013;13(1):36-44.

57. Bahrami E, et al. Leptin hormone level in serum of opticospinal, neuromyelitisoptica and multiple sclerosis patients. Clin Exp Neuroimmunol. 2014;5(1):77-83.
58. Galgani M, et al. Leptin Modulates the Survival of Autoreactive CD4+ T Cells through the Nutrient/Energy-Sensing Mammalian Target of Rapamycin Signaling Pathway. J Immunol. 2010;185(12):7474-9.

59. S. Emamgholipour, S. M. Eshaghi, A. Hossein-nezhad, K. Mirzaei, Z. Maghbooli, and M. A. Sahraian, "Adipocytokine profile, cytokine levels and Foxp3 expression in multiple sclerosis: a possible link to susceptibility and clinical course of disease," PLoS One, vol. 8, no. 10, 2013.

60. Evangelopoulos ME, Koutsis G, Markianos M. Serum leptin levels in treatment-naive patients with clinically isolated syndrome or relapsing-remitting multiple sclerosis. Autoimmune Dis. 2014;2014:1-6.

61. Rotondi M, et al. Severe disability in patients with relapsingremitting multiple sclerosis is associated with profound changes in the regulation of leptin secretion. Neuroimmunomodulation. 2013;20(6):341-7.

62. Messina $\mathrm{S}$, et al. Increased leptin and A-FABP levels in relapsing and progressive forms of MS. BMC Neurol. 2013;13(1):172.

63. Frisullo $\mathrm{G}$, et al. The effect of disease activity on leptin, leptin receptor and suppressor of cytokine signalling-3 expression in relapsing-remitting multiple sclerosis. J Neuroimmunol. 2007;192(1-2):174-83.

64. J. D. J. Guerrero-García, L. Carrera-Quintanar, R. I. López-Roa, A. L. Márquez-Aguirre, A. E. Rojas-Mayorquín, and D. OrtuñoSahagún, "Multiple sclerosis and obesity: possible roles of adipokines," Mediators of Inflammation, vol. 2016. 2016.

65. Reyes M, Quintanilla C, Burrows R, Blanco E, Cifuentes M, Gahagan S. Obesity is associated with acute inflammation in a sample of adolescents. Pediatr Diabetes. 2015;16(2):109-16.

66. Lord GM, Matarese G, Howard JK, Baker RJ, Bloom SR, Lechler RI. Leptin modulates the T-cell immune response and reverses starvation-induced immunosuppression. Nature. 1998;394(6696): 897-901.

67. Ochoa-Repáraz J, et al. Central nervous system demyelinating disease protection by the human commensal bacteroides fragilis depends on polysaccharide A expression. J Immunol. 2010;185(7)

68. Wu GD, et al. Linking long-term dietary patterns with gut microbial enterotypes. Science. 2011;334(6052):105-8.

69. W. J. van den Hoogen, J. D. Laman, and B. A. t'Hart, "Modulation of multiple sclerosis and its animal model experimental autoimmune encephalomyelitis by food and gut microbiota," Front. Immunol., vol. 8, no. 2017.

70. Voskuhl RR, Gold SM. Sex-related factors in multiple sclerosis susceptibility and progression. Nat Rev Neurol. 2012;8(5):255-63.

71. Le Chatelier E, et al. Richness of human gut microbiome correlates with metabolic markers. Nature. 2013;500:541-6. 